\title{
Transcatheter aortic valve implantation in the elderly: an umbrella review
}

\author{
Barbara Antonazzo1, Giuseppe Biondi-Zoccai ${ }^{2,3}$, Antonino G. M. Marullo1, Giacomo Frati ${ }^{1,4}$, Stefano \\ Ronzoni', Giovanni A. Chiariello ${ }^{5}$, Francesco Versaci ${ }^{6}$, Arturo Giordano $^{7}$ \\ 'Division of Geriatrics, Israelite Hospital, Rome 00148, Italy. \\ 2Department of Medico-Surgical Sciences and Biotechnologies, Sapienza University of Rome, Latina 04110, Italy. \\ ${ }^{3}$ Mediterranea Cardiocentro, Napoli 80122, Italy. \\ ${ }^{4}$ IRCCS NEUROMED, Pozzilli 86077, Italy. \\ Institute of Cardiology, Fondazione Policlinico Universitario A. Gemelli IRCCS, Università Cattolica del Sacro Cuore, Rome \\ 00168, Italy. \\ ${ }^{6}$ UOC UTIC Emodinamica e Cardiologia, S. Maria Goretti Hospital, Latina 04100, Italy. \\ ${ }^{7}$ Unità Operativa di Interventistica Cardiovascolare, Pineta Grande Hospital, Castel Volturno 81030, Italy.
}

Correspondence to: Prof. Giuseppe Biondi-Zoccai, Department of Medico-Surgical Sciences and Biotechnologies, Sapienza University of Rome, Corso della Repubblica 79, Latina 04100, Italy. E-mail: giuseppe.biondizoccai@uniroma1.it

\begin{abstract}
How to cite this article: Antonazzo B, Biondi-Zoccai G, Marullo AGM, Frati G, Ronzoni S, Chiariello GA, Versaci F, Giordano A Transcatheter aortic valve implantation in the elderly: an umbrella review. Vessel Plus 2020;4:3.

http://dx.doi.org/10.20517/2574-1209.2019.33
\end{abstract}

Received: 11 Dec 2019 First Decision: 30 Dec 2019 Revised: 11 Jan 2020 Accepted: 14 Jan 2020 Published: 12 Feb 2020

Science Editor: Mario F. L. Gaudino Copy Editor: Jing-Wen Zhang Production Editor: Tian Zhang

\begin{abstract}
Aim: The management of aortic stenosis has seen momentous changes thanks to the introduction of transcatheter aortic valve implantation (TAVI, i.e., transcatheter aortic valve replacement). Indications to TAVI have expanded progressively to intermediate- and low-risk patients, but trends in life expectancy have led to an increase of elderly but fit individuals with aortic stenosis eligible for TAVI.
\end{abstract}

Methods: We reviewed the current evidence base on TAVI in the elderly by conducting an umbrella review (i.e., overview of systematic reviews), based on a formal bibliographic search for systematic reviews on TAVI in elderly patients ( $\geq 65$ years). Key, study, patient, procedural, and outcome data were extracted, and validity formally appraised with the Oxman-Guyatt index.

Results: From 71 citations, eight reviews were included (totaling 39 studies and 8579 patients): five systematic reviews, and three meta-analyses. Topics of interest were cognitive function before and after TAVI, predictive role of muscle mass and frailty on post-TAVI outcomes, comparative safety and effectiveness of TAVI, and role of rehabilitation to improve patient outlook after TAVI. Thirty-three additional studies were retrieved by means of snowballing, emphasizing the 
role of multidimensional assessment of elderly patients scheduled for TAVI, in order to maximize its appropriateness, effectiveness, and safety.

Conclusion: It is crucial to consider frailty scores, as well as nutrition and functional status, in addition to established surgical risk scores, in elderly patients considered for TAVI to improve risk prediction, reinforcing the favorable impact of this therapy to improve cognitive function.

Keywords: Aortic stenosis, elderly, transcatheter aortic valve implantation, transcatheter aortic valve replacement

\section{INTRODUCTION}

Surgical aortic valve replacement (SAVR) has been for several decades the default management strategy for severe aortic stenosis in fit patients ${ }^{[1]}$. However, an ever increasing elderly population, often fraught with substantial comorbidities, has challenged in many cases the risk-benefit profile of surgery ${ }^{[2]}$. Accordingly, less invasive strategies were developed, including balloon aortic valvuloplasty ${ }^{[3]}$.

Building upon developments in materials and procedures, and inspired by breakthrough results of stenting for coronary and endovascular procedures, transcatheter aortic valve implantation (TAVI), also called transcatheter aortic valve replacement (TAVR), was introduced by Alain Cribier almost two decades ago ${ }^{[4-6]}$. The successes of TAVI have been dramatic indeed, as poignantly summarized by the recent US Food and Drug Administration approval of new-generation devices for TAVI even in patients at low surgical risk ${ }^{[7]}$. However, TAVI continues to be considered and used mostly for elderly patients, given the uncertainty on long-term and very long-term device durability ${ }^{[8]}$.

Despite the evidently favorable risk-benefit profile of TAVI in general, and in the elderly in particular, several areas of investigation and debate persist, typically focusing on indication, timing, procedural aspects, device choice, ancillary medical management, and post-procedural results ${ }^{[6]}$. We aimed at exploiting the synthesizing power of umbrella review studies to reconcile conflicting sources of evidence on TAVI in the elderly, in order to inform current practice and guide future research ${ }^{[9]}$.

\section{METHODS}

This scoping umbrella review was conducted in keeping with best practice recommendations, and reported accordingly ${ }^{[9]}$. Specifically, we used a multifaceted approach for evidence accrual, avoiding a specific or restrictive definition of elderly. First, PubMed was searched using the following string: "\{elderly OR octogenarian* OR octagenarian* OR nonagenarian* OR old OR aged OR [age AND (advanced OR old)]\} AND transcatheter AND aortic AND valve AND (implantation OR replacement) AND systematic[sb]" up to 31 October 2019. Accordingly, any review detailing on, at least in part, nonagenarians, octogenarians, aged patients, or subjects with advanced or old age could be included, provided it also focused on TAVI. Thereafter, we used backward and forward snowballing to identify additional citations. Afterwards, potentially relevant citations were screened at the title/abstract level. Potentially relevant hits were then retrieved as full-texts.

We included systematic reviews (i.e., overviews of published clinical studies including two or more primary original reports) detailing TAVI in elderly patients (defined as people aged $\geq 65$ ), irrespective of their focus on diagnosis, prognosis, device choice, procedural aspects, or outcomes, to avoid being overly restrictive. Several domains were abstracted, including review features, study aspects, and other details on included patients, procedures, and outcomes. Review validity was appraised with the Oxman and Guyatt Overview Quality Assessment Questionnaire ${ }^{[10]}$. All reviewing activities were performed by two independent reviewers, with divergences solved after consensus. 
Table 1. Included systematic reviews on TAVI in the elderly

\begin{tabular}{|c|c|c|c|c|c|}
\hline Ref. & PubMed ID & Focus & Studies & Patients & Highlights \\
\hline Anand et al. ${ }^{[11]}$ & 28927173 & Frailty & 10 & 4592 & Frailty is a significant predictor of adverse events after TAVI \\
\hline Fink et $a .^{[12]}$ & 26192563 & Cognitive function & 1 & 64 & Cognitive function may be impaired after TAVI \\
\hline Furukawa et al. ${ }^{[13]}$ & 25916404 & Frailty & 6 & 1023 & Frailty is a significant predictor of adverse events after TAVI \\
\hline Lai et al. ${ }^{[14]}$ & 25785192 & Cognitive function & 6 & 349 & Cognitive function remains stable or improves after TAVI \\
\hline Mohammadi et al. ${ }^{[15]}$ & 26728319 & Effectiveness of TAVI & NA & NA & $\begin{array}{l}\text { TAVI impacts favorably on morbidity and mortality in elderly } \\
\text { patients with AS }\end{array}$ \\
\hline Ribeiro et al. ${ }^{[16]}$ & 28071146 & Rehabilitation & 5 & 292 & $\begin{array}{l}\text { Cardiac rehabilitation improves functional capacity and QoL } \\
\text { after TAVI }\end{array}$ \\
\hline Sepehri et $a^{[17]}$ & 25199821 & Frailty & 3 & 378 & Frailty is a significant predictor of adverse events after TAVI \\
\hline Soud et al. ${ }^{[18]}$ & 30915667 & Muscle mass & 8 & 1881 & $\begin{array}{l}\text { Skeletal muscle area appraised with CT is a significant } \\
\text { predictor of adverse events after TAVI }\end{array}$ \\
\hline
\end{tabular}

CT: computed tomography; NA: not applicable; QoL: quality of life; TAVI: transcatheter aortic valve implantation; AS: aortic stenosis

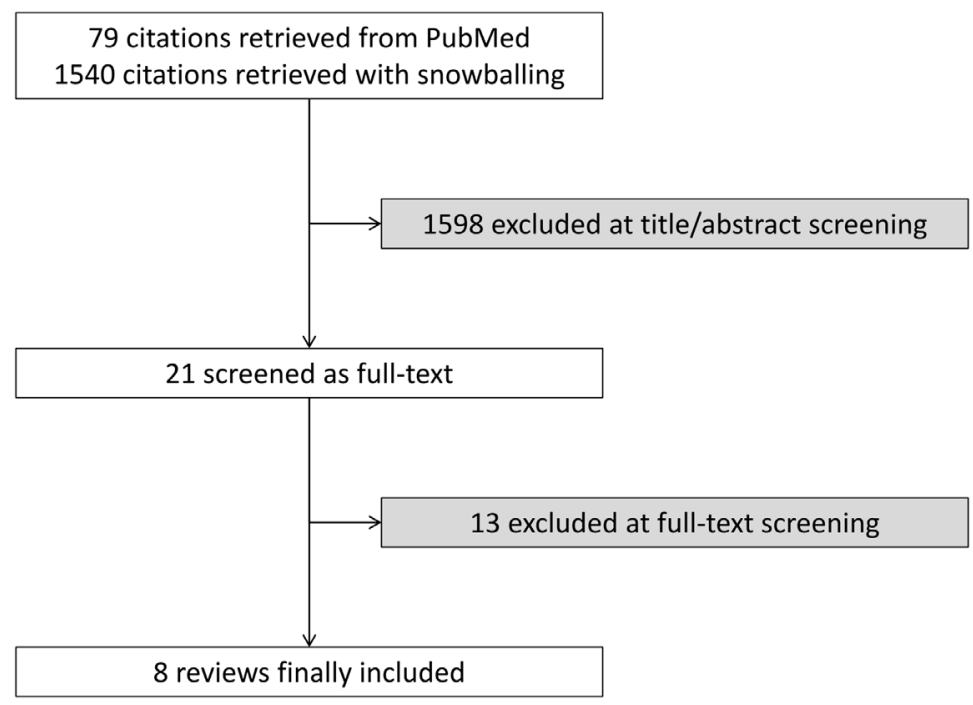

Figure 1. Review profile, detaling study search and selection

\section{RESULTS}

From an initial set of 1619 citations, a subset of 21 were retrieved as full-texts, finally yielding eight reviews, totaling 39 primary studies and 8579 patients [Table 1 and Figure 1] ${ }^{[1-18]}$. Five were systematic reviews only, and the remaining three also provided meta-analysis results ${ }^{[11,16,18]}$. The topics of interest were cognitive function before and after TAVI ${ }^{[12,14]}$, predictive role of muscle mass and frailty on post-TAVI outcomes ${ }^{[11,13,17,18]}$, comparative safety and effectiveness of TAVI ${ }^{[15]}$, and role of rehabilitation to improve patient outlook after TAVI ${ }^{[16]}$. Review quality ranged from high validity and low risk of bias for five reviews $^{[11,12,16-18]}$, to low validity and high risk of bias in three reviews ${ }^{[13-15]}$ [Table 2], with lack of adequate reporting being the most common limitation.

In particular, Anand et al. ${ }^{[1]]}$ performed a systematic review and meta-analysis appraising the prognostic impact of frailty in patients undergoing TAVI, including a total of 10 studies and 4592 patients. They concluded that frailty proved to be a significant predictor of adverse events after TAVI. Similar findings were reported by prior reviews such as the systematic review conducted by Furukawa et al. ${ }^{[13]}$, encompassing six primary studies and 1023 patients, and the one authored by Sepehri and colleagues, totaling three studies and 378 subjects $^{[17]}$. A relatively similar focus was chosen by Soud et al. ${ }^{[18]}$, who pooled eight studies including 1881 to appraise the predictive usefulness of appraising skeletal muscle mass by means of computed tomography (CT). CT-derived muscle area showed a significant prognostic 


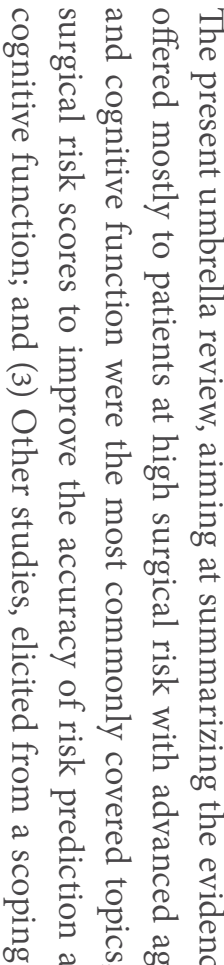

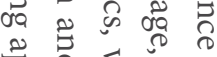

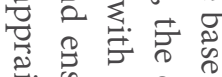

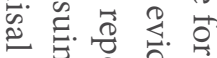

o o o :

दे के के

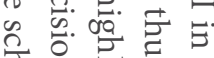

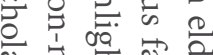

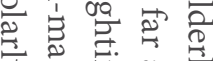

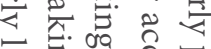

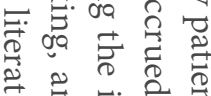

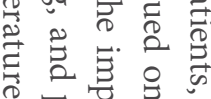

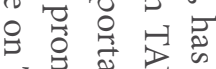

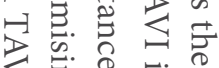

క.

ज्ञ

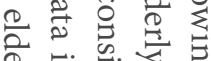

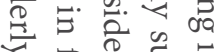

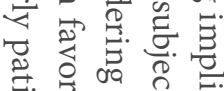

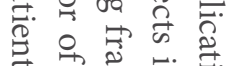
क人

ㅌ..ㄴ.

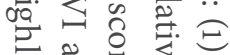

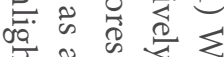

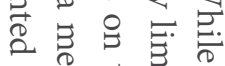

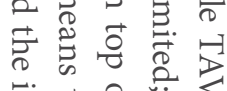

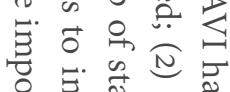

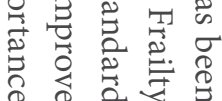

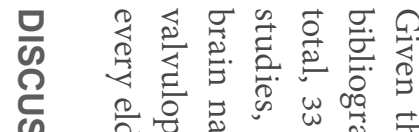

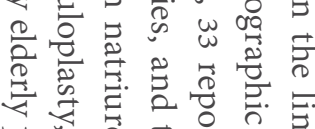

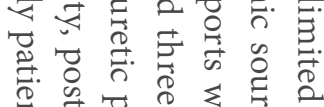

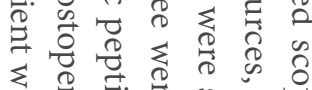
装.

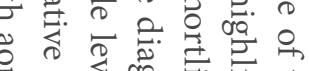
. 을

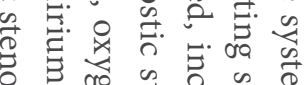

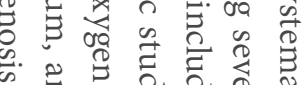

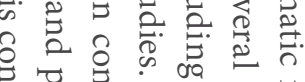

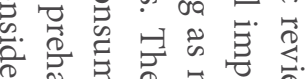

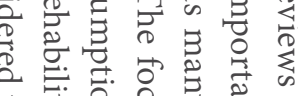

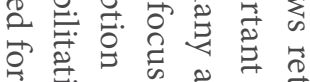

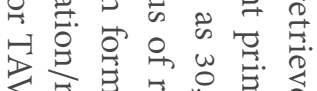

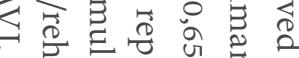
एँ 랄

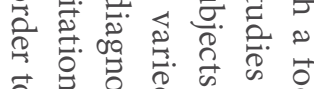
००

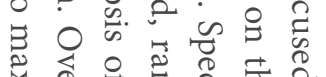

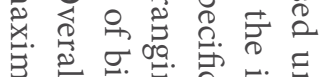

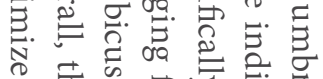

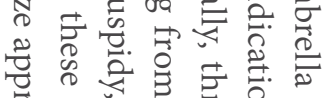

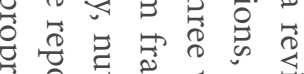

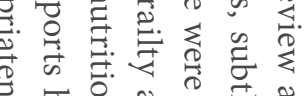

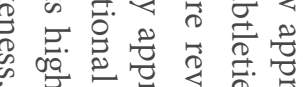

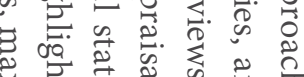

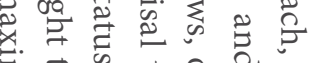

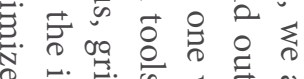

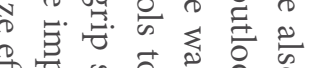

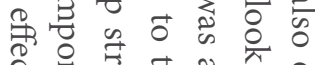

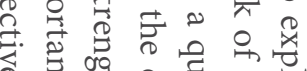

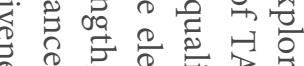
尊 을 列:

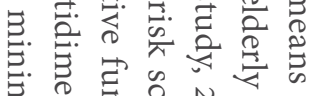

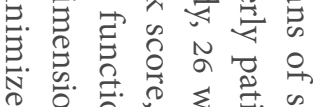

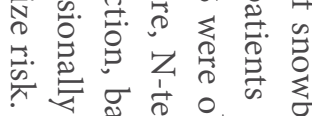

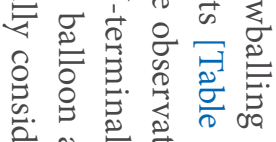

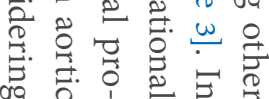

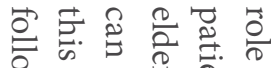

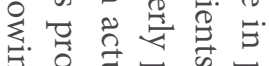

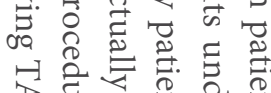

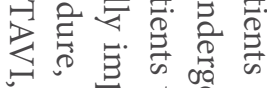

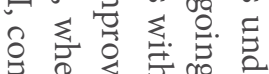
है ङ

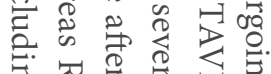
它

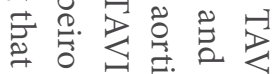
ㄹ. 울 क.

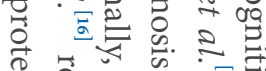

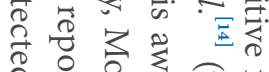

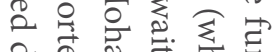
‥ 율

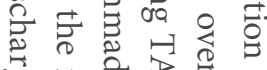

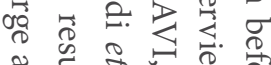

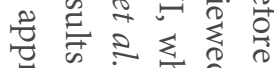

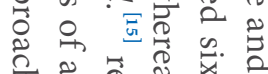
곤. क्ष

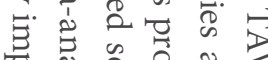

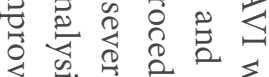
ठ․

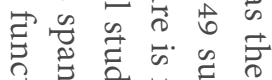

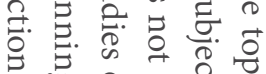

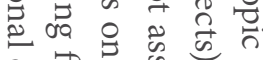
要

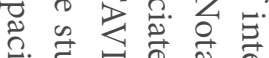

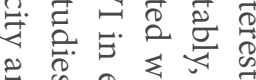

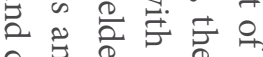

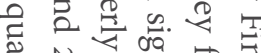

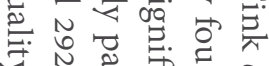

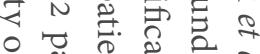

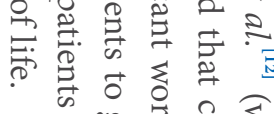

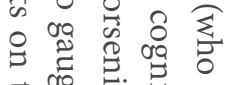

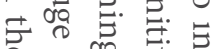
点

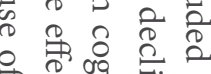

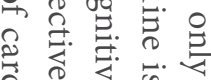

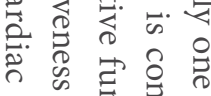

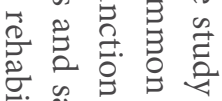

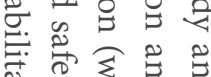

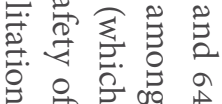

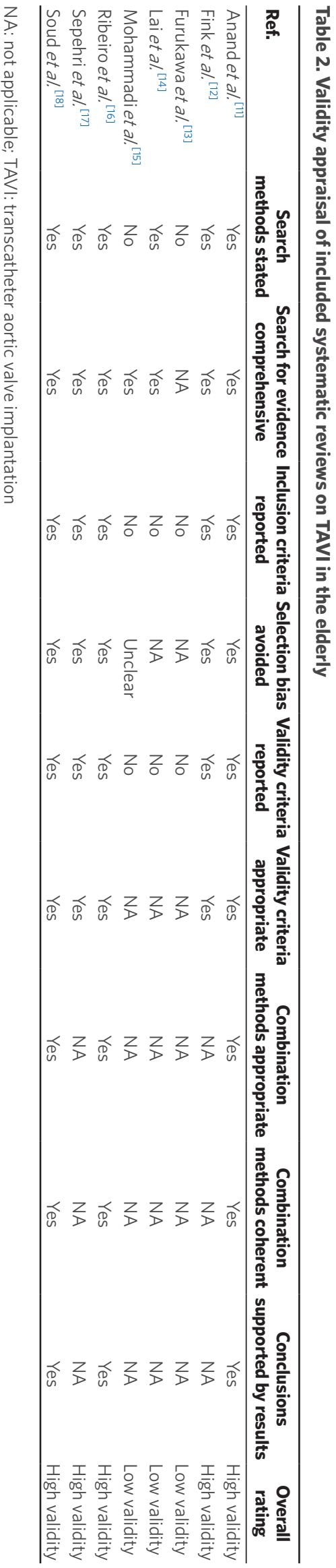




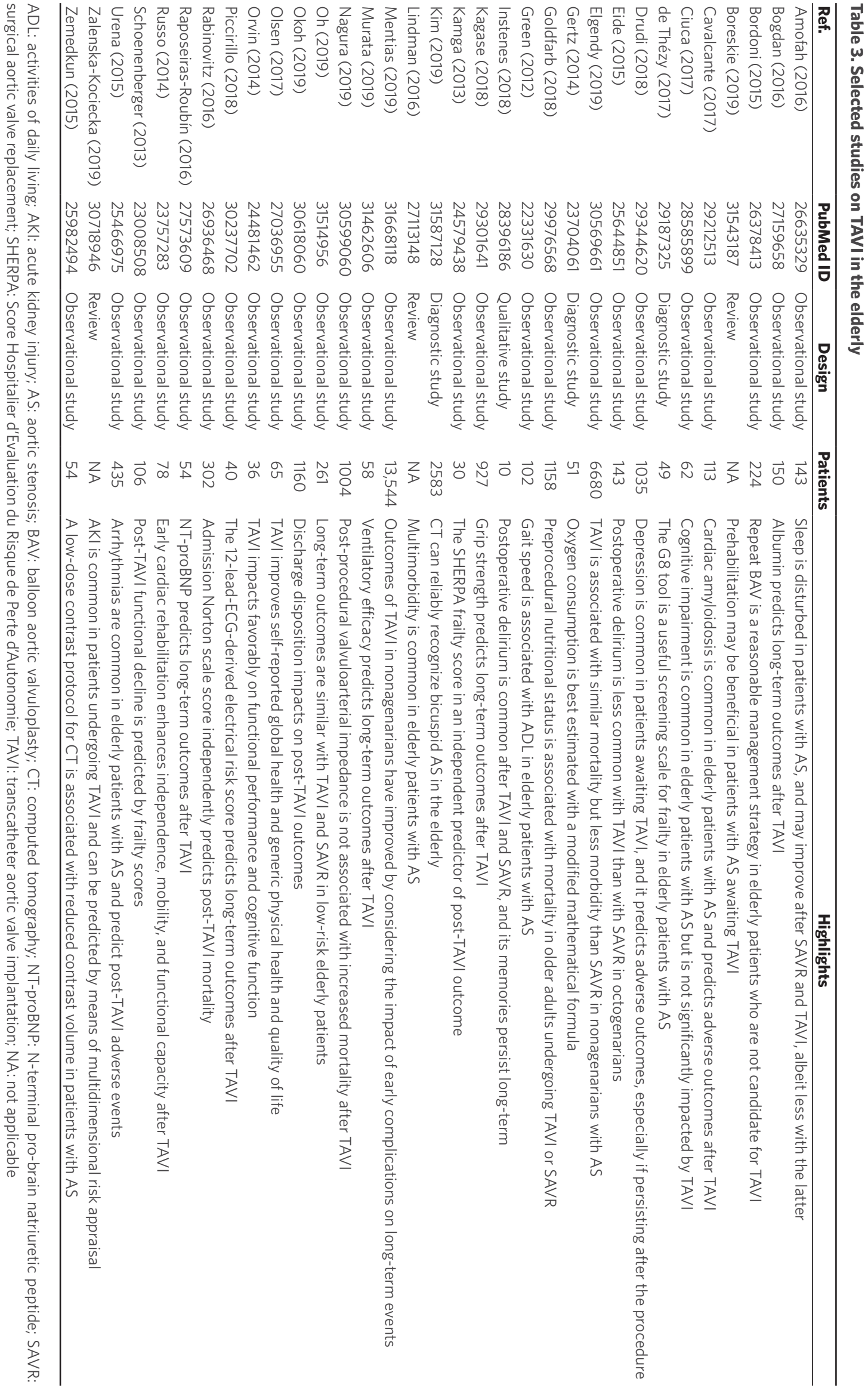


of multidimensional appraisal and management of these subjects, while confirming the promising role of TAVI in comparison to medical therapy, balloon aortic valvuloplasty, and SAVR in elderly patients.

The evolution of TAVI has been momentous, and, since the first pioneering cases, TAVI is challenging the role of SAVR even in low-risk patients ${ }^{[6]}$. These successes depend on major refinements in diagnostic tools (e.g., CT angiography for precise sizing), patient preparation, device improvements, ancillary management approaches, and post-procedural management ${ }^{[19-26]}$. These refinements and the fact that TAVI was initially validated in trials enrolling mostly high-risk patients with advanced age would suggest that all major issues concerning TAVI in the elderly have been solved ${ }^{[6]}$. This is of course false, and substantial research is still ongoing on several related topics. For instance, the aspects of cost utility and futility remain actively debated, as well as all issues pertinent to patient preparation, device selection, predilation vs. postdilation, embolic protection, and post-procedural antithrombotic therapy ${ }^{[6,11-15,27-30]}$.

The present umbrella review, albeit limited in comparison to other umbrella reviews authored by our research group given the limited scope of the available evidence base, highlights the importance of frailty assessment to predict short-term complications and long-term results of TAVI in the elderly, the emerging role of cognitive assessment before TAVI and prevention of cognitive decline due to TAVI complications, and the usefulness of cardiac rehabilitation in all old patients with severe aortic stenosis undergoing TAVI. Further evidence highlights the importance of assessing in a multidimensional fashion the presence of comorbidities, nutritional status, grip strength, gait speed, and overall functional status, while confirming the favorable clinical performance at short- and mid-term follow-up of TAVI, without discounting the niche role of balloon aortic valvuloplasty in patients at prohibitive risk, and the pivotal function of SAVR in fit patients.

Limitations of this umbrella review are of course those typical of overviews of reviews, including the risk of ecological fallacy ${ }^{[9]}$. In addition, while studies on TAVI usually enroll mostly patients with advanced age, only a limited set of systematic reviews explicitly aimed at the topic of TAVI in the elderly. Accordingly, further reviews are eagerly awaited to more poignantly summarize the evidence base for this important topic in structural heart disease. Focusing on the definition of elderly, our definition of elderly as aged $\geq 65$ years is quite arbitrary, especially in the context of TAVI, which is often performed in much older subjects $^{[31,32]}$. However, this remains a common pragmatic definition for many patients, non-specialists, and decision-makers ${ }^{[32]}$. In addition, by default, umbrella reviews have limited room to select primary studies from included reviews. Similarly, having an unrestrictive approach at TAVI indication (e.g., stenosis, regurgitation, and valve-in-valve) risks mixing "apples with oranges" and providing overly heterogenous results. Most importantly, the TAVI landscape continues to change, shifting from prohibitive and high-risk patients, to subjects at intermediate or low risk. Another crucial evolution has centered on devices, which evolved from the crude Cribier-Edwards device to current-generation, low-profile and fully repositionable/ retrievable ones ${ }^{[21]}$. However, as stated above, by definition, umbrella reviews cannot limit inclusion to a given group of primary studies. Accordingly, we can only let readers subset the included systematic reviews/studies according to the specific features they are most interested in, when wishing to apply to specific patient subgroups the findings of our umbrella review.

In conclusion, the scholarly literature on TAVI continues to accrue, reaffirming the favorable risk-benefit balance of this breakthrough technology in patients with severe aortic stenosis, including selected lowrisk subjects. Our umbrella review, including eight systematic reviews, 39 primary studies, and 8579 patients, highlights the importance of considering frailty scores, as well as nutrition and functional status, in addition to established surgical risk scores in elderly patients considered for TAVI to improve risk prediction, reinforcing the favorable impact of this therapy to improve cognitive function. 


\section{DECLARATIONS}

\section{Authors' contributions}

Designed the review, performed all reviewing activities and drafted the manuscript: Antonazzo B, Biondi-

Zoccai G

Participated in review design, supervised all reviewing activities, and provided critical contributions to the manuscript: Marullo AGM, Frati G, Ronzoni S, Chiariello GA, Versaci F, Giordano A

All authors eventually approved it in its final version.

\section{Availability of data and materials}

Not applicable.

\section{Financial support and sponsorship}

None.

\section{Conflicts of interest}

All authors declared that there are no conflicts of interest.

\section{Ethical approval and consent to participate}

Not applicable.

\section{Consent for publication}

Not applicable.

\section{Copyright}

(c) The Author(s) 2020.

\section{REFERENCES}

1. Harris AW, Pibarot P, Otto CM. Aortic stenosis: guidelines and evidence gaps. Cardiol Clin 2020;38:55-63.

2. Kwiecień A, Hrapkowicz T, Filipiak K, Przybylski R, Kaczmarczyk M, et al. Surgical treatment of elderly patients with severe aortic stenosis in the modern era - review. Kardiochir Torakochirurgia Pol 2018;15:188-95.

3. Baber U, Kini AS, Moreno PR, Sharma SK. Aortic stenosis: role of balloon aortic valvuloplasty. Cardiol Clin 2013;31:327-36.

4. Cribier A, Eltchaninoff H, Bash A, Borenstein N, Tron C, et al. Percutaneous transcatheter implantation of an aortic valve prosthesis for calcific aortic stenosis: first human case description. Circulation 2002;106:3006-8.

5. Bohula May EA, Faxon D. Transcatheter aortic valve replacement: history and current status. Trends Cardiovasc Med 2013;23:172-8.

6. Giordano A, Biondi-Zoccai G, Frati G. Transcatheter Aortic Valve Implantation: Clinical, Interventional and Surgical Perspectives. Cham: Springer Nature Publishing; 2019.

7. Boskovski MT, Nguyen TC, McCabe JM, Kaneko T. Outcomes of transcatheter aortic valve replacement in patients with severe aortic stenosis: a review of a disruptive technology in aortic valve surgery. JAMA Surg 2019; Epub ahead of print [DOI: 10.1001/ jamasurg.2019.4449]

8. Adams HSL, Ashokkumar S, Newcomb A, MacIsaac AI, Whitbourn RJ, et al. Contemporary review of severe aortic stenosis. Intern Med J 2019;49:297-305.

9. Biondi-Zoccai G. Umbrella Reviews: Evidence Synthesis with Overviews of Reviews and Meta-Epidemiologic Studies. Cham: Springer International Publishing; 2016.

10. Oxman AD, Guyatt GH.Validation of an index of the quality of review articles. J Clin Epidemiol 1991;44:1271-8.

11. Anand A, Harley C, Visvanathan A, Shah ASV, Cowell J, et al. The relationship between preoperative frailty and outcomes following transcatheter aortic valve implantation: a systematic review and meta-analysis. Eur Heart J Qual Care Clin Outcomes 2017;3:123-32.

12. Fink HA, Hemmy LS, MacDonald R, Carlyle MH, Olson CM, et al. Intermediate- and long-term cognitive outcomes after cardiovascular procedures in older adults: a systematic review. Ann Intern Med 2015;163:107-17.

13. Furukawa H, Tanemoto K. Frailty in cardiothoracic surgery: systematic review of the literature. Gen Thorac Cardiovasc Surg 2015;63:425-33.

14. Lai KS, Herrmann N, Saleem M, Lanctôt KL. Cognitive outcomes following transcatheter aortic valve implantation: a systematic review. Cardiovasc Psychiatry Neurol 2015;2015:209569.

15. Mohammadi M, Hill C, Chaney S. Is transcatheter aortic valve replacement a safe treatment for elderly patients with severe aortic valve 
stenosis? J Am Assoc Nurse Pract 2016;28:387-92.

16. Ribeiro GS, Melo RD, Deresz LF, Dal Lago P, Pontes MR, et al. Cardiac rehabilitation programme after transcatheter aortic valve implantation versus surgical aortic valve replacement: Systematic review and meta-analysis. Eur J Prev Cardiol 2017;24:688-97.

17. Sepehri A, Beggs T, Hassan A, Rigatto C, Shaw-Daigle C, et al. The impact of frailty on outcomes after cardiac surgery: a systematic review. J Thorac Cardiovasc Surg 2014;148:3110-7.

18. Soud M, Alahdab F, Ho G, Kuku KO, Cejudo-Tejeda M, et al. Usefulness of skeletal muscle area detected by computed tomography to predict mortality in patients undergoing transcatheter aortic valve replacement: a meta-analysis study. Int J Cardiovasc Imaging 2019;35:1141-7.

19. Graziani F, Manfredonia L, Locorotondo G, Burzotta F, Trani C. When is compassionate appropriate for end-stage aortic valve stenosis? Minerva Cardioangiol 2018;66:221-2.

20. Zhang X, Wang T, Lan R, Dai Q, Kang L, et al. Meta-analysis comparing results of transcatheter versus surgical aortic-valve replacement in patients with severe aortic stenosis. Am J Cardiol 2020;125:449-58.

21. Gatto L, Biondi-Zoccai G, Romagnoli E, Frati G, Prati F, et al. New-generation devices for transcatheter aortic valve implantation. Minerva Cardioangiol 2018;66:747-61.

22. Spaccarotella C, Mongiardo A, De Rosa S, Indolfi C. Transcatheter aortic valve implantation in patients at intermediate surgical risk. Int J Cardiol 2017;243:161-8.

23. Presbitero P, Iannetta L, Pagnotta P, Reimers B, Rossi ML, et al. Transcatheter aortic valve implantation in bicuspid anatomy: procedural results with two different types of valves. Minerva Cardioangiol 2018;66:129-35.

24. Repossini A, Fischlein T, Solinas M, DI Bacco L, Passaretti B, et al. Stentless sutureless and transcatheter valves: a comparison of the hemodynamic performance of different prostheses concept. Minerva Cardioangiol 2018;66:180-90.

25. Santarpino G, Gregorini R, Specchia L, Albano A, Nicoletti A, et al. Sutureless aortic valve replacement vs. transcatheter aortic valve implantation: a review of a single center experience. Minerva Cardioangiol 2018;66:160-2.

26. Mishra S. Will percutaneous valves replace the surgical valves: Another one bites the dust? Indian Heart J 2016;68:249-51.

27. Giordano A, Corcione N, Ferraro P, Bedogni F, Testa L, et al; RISPEVA (Registro Italiano GISE sull'impianto di Valvola Aortica Percutanea) Study Investigators. Outcome of patients undergoing transcatheter aortic valve implantation after prior balloon aortic valvuloplasty. J Invasive Cardiol 2018;30:380-5.

28. Murdoch DJ, Webb JG, Ye J, Sathananthan J, Hensey M, et al. Transcatheter aortic-valve replacement - 10 years later. N Engl J Med 2019;380:1773-4.

29. Giordano A, Corcione N, Ferraro P, Pieri P, Avellino R, et al. Propensity-score-adjusted comparison of Evolut vs. Portico devices for transcatheter aortic valve implantation. J Cardiovasc Med (Hagerstown) 2019;20:351-7.

30. Giordano A, Corcione N, Ferraro P, Morello A, Conte S, et al; Registro Italiano GISE sull'impianto di Valvola Aortica Percutanea (RISPEVA) Study Investigators. Comparative one-month safety and effectiveness of five leading new-generation devices for transcatheter aortic valve implantation. Sci Rep 2019;9:17098.

31. Orimo H. Reviewing the definition of elderly. Nihon Ronen Igakkai Zasshi 2006;43:27-34.

32. World Health Organization: Proposed working definition of an older person in Africa for the MDS Project. Available from: https://www. who.int/healthinfo/survey/ageingdefnolder/en/ [Last accessed on 17 Jan 2020] 